\title{
Synthetic cannabinoids and renal injury
}

\author{
Alper Alp \\ Department of Nephrology, Van Training and Research Hospital, Van, Turkey
}

\section{Dear Editor;}

I think that review article entitled "Synthetic Cannabinoids" written by Bilici, and published in Vol 12, No.2 pp. 121-6 of your periodical conveys importance in that it emphasizes the awareness concerning a clinical condition whose incidence is increasing as a current, and potential dangerous addiction [1].

Unfortunately, rapidly increasing addiction to narcotics, and synthetic illicit drugs especially among young population has currently perhaps become one of the most important social, and medical problematic issues both in the world, and in our country. Acute kidney injury (AKI) is one of the serious complications in clinical practice because of ensuing higher mortality, and morbidity rates, and cost. It has quite a long list of etiological factors. Among addictive substances, destructive effects of heroin, and cocaine on renal tissues are very well known, and they have taken their place in classical textbooks. Especially in recent years, some novel synthetic chemical compounds with increasingly widespread use have been introduced into black market. Synthetic cannabinoids (SCs) constitute an important group among them. Cases with renal injury developed related to the use of SCs have been described, and detected at a rapidly increasing rate. Though very rarely seen, these substances have adverse [2] renal effects as hyperkalemia, and hypertension apart from AKI. Lit- erature reviews have revelaed that $\mathrm{SC}$-related cases with AKI were mostly of prerenal AKIs, and due to rapid improvement in renal functions, many of them recovered with general supportive treatment without renal biopsy. However in some cases the need for transient hemodialysis, and steroid treatment arises. This prerenal condition where prominent symptoms of nausea, vomiting, abdominal pain are associated with chronic SC use is called cannabinoid hyperemesis syndrome. Rhabdomyolisis is another etiological factor for acute renal injury. In these patients levels of creatinine kinase, myoglobulin, and uric acid should be measured on admission. Its clinical picture does not differ from classical manifestations, and generally the patients are treated without the need for renal replacement therapies. In rare cases who underwent renal biopsies, the most frequently encountered histopathological finding is acute tubular necrosis (ATN). Despite presence of histopathological findings in renal biopsy, some patients do not present with classical ATN clinic which suggests the involvement of different pathophysiologic mechanisms. Besides, rarely, interstitial nephritis, and cresentic glomerulonephritis can be seen.

Especially in young patients with acute renal injury but without any known renal disease who presented to the emergency services, with a history of substance use, seizures or blurred consciousness, use of synthetic substances) should be suspected. 
Adverse renal effects of these substances in addition to their central, and psychiatric effects should be recognized. Even though all components of these substances can not be identified, toxicological analyses of urine samples should be performed. I thank Bilici, MD, $\mathrm{PhD}$, and your scientific journal for this detailed review article.

\section{REFERENCES}

1. Bilici R. Synthetic cannabinoids. North Clin Istanbul 2014;1:121-6.

2. Centers for Disease Control and Prevention (CDC). Acute Kidney Injury Associated with Synthetic Cannabinoid Use Multiple States, 2012. Morbidity and Mortality Weekly Report (MMWR) 2013;62:93-8. 